\title{
A Potential Yeast Strains for Biological Control of Mosquitoes
}

\author{
D Satwika*1, V R A Permatasari ${ }^{1}$, G E N Cahyani ${ }^{1}$ \\ ${ }^{1}$ Faculty of Biotechnology, Universitas Kristen Duta Wacana, Jl. dr. Wahidin 5-25, Yogyakarta 55224, Indonesia \\ *Corresponding author: dhira@staff.ukdw.ac.id
}

\begin{abstract}
Malaria is one of the diseases that have not been successfully eradicated, and are still a threat in many countries. Efforts have been made to eradicate vector-borne diseases, including the use of insecticides and physical approaches, which are proven not effective in overcoming the malaria problem. One of the recent approach to overcome the problem is employing endosymbiotic microorganisms for killing the mosquitoes as malaria vector. Antagonistic yeast that inhibit the growth of other organisms has been reported from various habitats, including the digestive tract of insects. It is then the purpose of this study to isolate potential yeast(s) as an antagonistic yeast from the digestive tract of mosquitoes. Local mosquitoes were used as samples, dissected and a serial platting was done for isolating the yeast. The obtained isolate(s) were tested for its antagonistic activity against a sensitive strain, Saccharomyces cerevisiae CEN-PK2-1C, and resulting in two isolates which showed consistent antagonistic activities. Molecular identification were done by employing the Internal Transcription Spacer (ITS) area as a marker gene. An isolate, namely NY24 showed the highest similarity with Jaminaea sp, while the NY38 isolate is located in the same cluster with Candida sp group. However, it is noteworthy to mention that both isolates are separated from what is known as VLE-yeast killer group, and deserve further research as potential candidates for mosquitoes' biocontrol agent, especially in Indonesia.
\end{abstract}

Keywords: antagonistic yeast, insects, Candida sp, Jaminaea sp

\section{INTRODUCTION}

Vector-borne diseases (VBD) as a result of insect activities, especially arthropods, like malaria, chikungunya, dengue, zika and HIV are still becoming the major threat for human health, not only for tropical or sub-tropical countries, but already becoming global issue [1]. Efforts have been done to fight these VBD, and it is proven physical and chemical approaches are not able to stop the spread of the diseases; global warming, travelling, including trading are some factors that favor the growth of mosquitoes which could carry pathogenic agents. On the other hand, malaria is still one of the most deadly disease, consider as world's most important vector-borne human diseases [1].
Considering that mosquitoes is an example of species that successfully evolve at diverse environment, a new approach should be taken in order to control the growth of this species. It is reported that the presence of microbiota in their body might be a key to do it, and a new approach called symbiotic control is introduced, which basically make use the presence of endosymbiont in the mosquitoes' body [2]. An example of this approach is the application of a strain of Serratia marcescens isolated from Anopheles sp that grow on the gonads and able to pass to the mosquitoes' intestine, and stay for generations. The presence of this bacteria will resulted in the inhibition of Plasmodium falciparum development in mosquitoes [3]. 
It has been reported that a yeast strain, called Wickerhamomyces anomalus lived in the mosquitoes' intestine, isolated from Anopheles stephensi. This yeast showed inhibitory effect to the Plasmodium $\mathrm{sp}$ either in vitro or in vivo, and showed a potential killing ability against the mosquitoes [4, 5, 6]. This so-called killer yeast could be recovered from diverse habitats and

\section{MATERIALS AND METHODS}

\subsection{Isolation of yeasts from mosquitoes}

Mosquito samples, Anopheles sp and Culex sp, were collected from nature in the laboratory of Universitas Kristen Duta Wacana, Yogyakarta by setting simple catchment. The insect digestive tract was then dissected, put in $\mathrm{NaCl}$ solution and platted on Yeast Peptone Dextrose (YPD) medium. Yeast isolation and the enrichment method were adapted basically according to previously published paper [8].

\subsection{Isolation and screening}

Colonies were screened mainly based on their macroscopic and microscopic observation. All of the yeast colonies were tested for their antagonistic activity by mean of eclipse assay and/or diffusion assay against the sensitive yeast Saccharomyces cerevisiae CEN.PK2-1c. The antagonistic yeasts are

\section{RESULTS AND DISCUSSION}

A total number of 80 mosquitoes were collected and samples from the digestive tract were used for yeast isolation, as can be seen in Figure 1. After

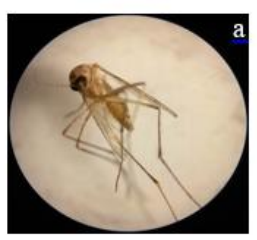

already reported elsewhere. These yeasts are taxonomically diverse, and targeting not only yeast, but could also be bacteria and protozoa [7]. Considering this background, it is then the aim of this research to isolate and screened antagonistic yeast(s) from local mosquitoes, and molecularly identify them.

incubated for 24 hours before the antagonistic activity assay, based on published paper [8].

\subsection{DNA isolation}

Total yeast DNA isolation was done for colonies showing the positive reaction or are able to inhibit the growth of the sensitive strain. The isolation methods adapted from published paper [9].

\subsection{DNA sequencing and analysis}

The DNA sequencing was done at PT. Genetika Science Indonesia, Jakarta, employing ITS as the genetic marker. Once the sequence(s) obtained, it is then compared to the online genetic database by conducting BLAST analysis (ncbi.nlm.nih.gov/BLAST). Multiple alignment of nucleotide sequences was done using ClustalX [10], and the phylogenetic tree was calculated by mean of MEGA5 with Maximum-Likelihood method and a bootstrap value of 1000 [11].

several dilution and isolation steps, a total of 14 yeast colonies were obtained, and some are presented in Figure 2.

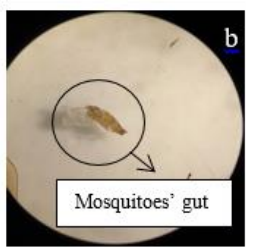

Figure 1. a) Mosquitoes samples used in this study, showing b) the digestive tract (transparent).

Those isolates were randomly chosen based on their macroscopic appearance, and subsequently observed under the microscope. They are distinguished based on the cell shape, size, color, as well as their specific structure, such as budding type. An example of the budding types could be $\mathrm{y}$. seen in Figure 3, showing uni- and bipolar budding [12]. A total of 13 different isolates were recognized that showed inhibition area on agar. Based on their morphological characteristics and initial antagonistic assay, 2 isolates were randomly chosen, namely NY24 and NY38, respectivel 

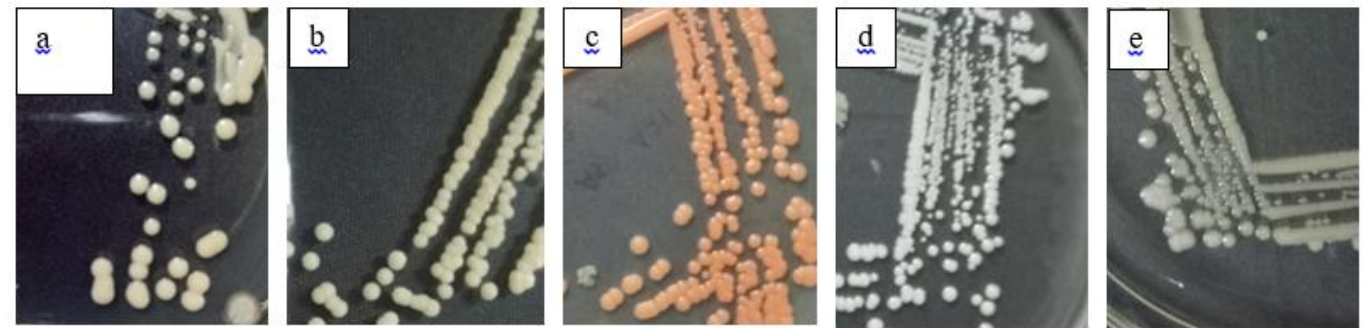

Figure 1. The pictures shows different types of yeast colonies that are recovered from the mosquitoes. a) isolate NY24 with big-round shape and white-dull color, b) isolate NY25 with small-round shape and white-dull color, c) isolate NY26 has a shiny-red-orange color and big-round shape, d) isolate NY27 with a tiny-round shape and white-dull color, and e) isolate NY38 with white and shiny color.

The 2 isolates were then checked for their antagonistic activity against the sensitive strain, and continued for another assay to confirm their killing ability. All of the tested isolates consistently showed the inhibition zone when tested against $S$. cerevisiae CEN.PK2-1c as can be seen in Figure 4.

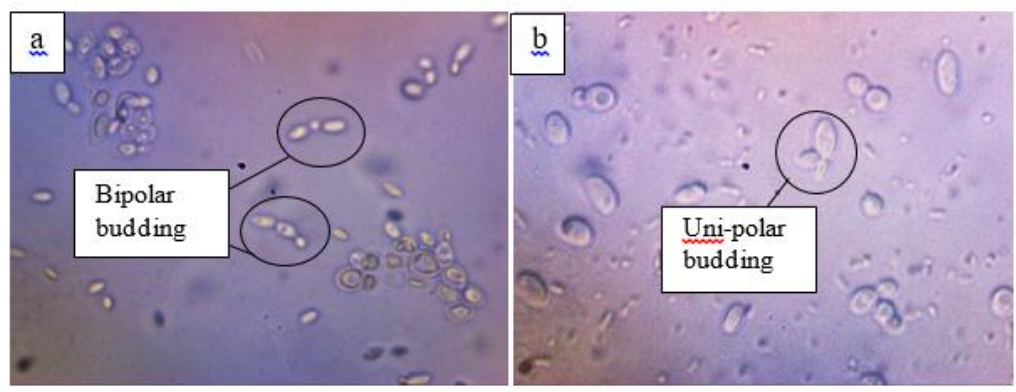

Figure 2. Microscopic morphology of isolate NY24 showing a) bipolar budding and b) isolate NY38 with a unipolar budding, magnification 10x100.

Researches have already reported the occurrence of yeasts that are able to exert antagonistic activity in diverse habitats [7, 13], with the need for nutrients as one of the most important factors. Many of these antagonistic activities are
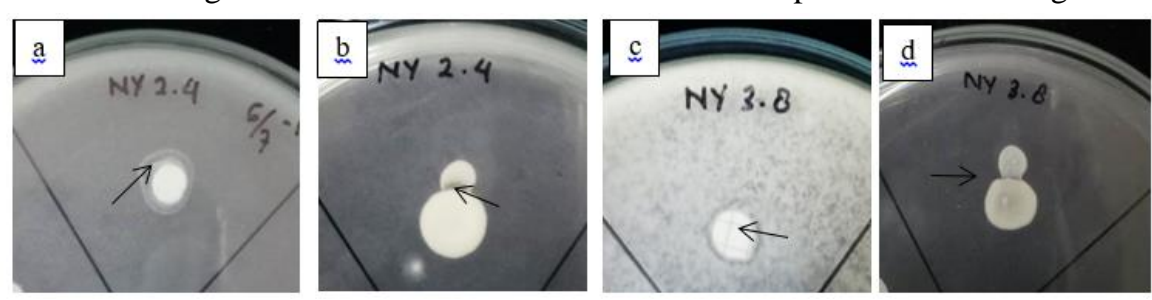

driven by extra-chromosomal genetic material in yeast, known as virus-like element (VLE) [14]. Therefore, the study was continued to analyze the molecular trait of the isolates obtained, and the results are presented in the Figure 5 below.

Figure 3. Images showing antagonistic ability of NY24 against $S$. cerevisiae CEN.PK2-1c on a) diffusion assay and b) eclipse assay; while c) result for isolate NY38 on diffusion assay and d) for eclipse assay (d), showing positive result against the sensitive strain. 


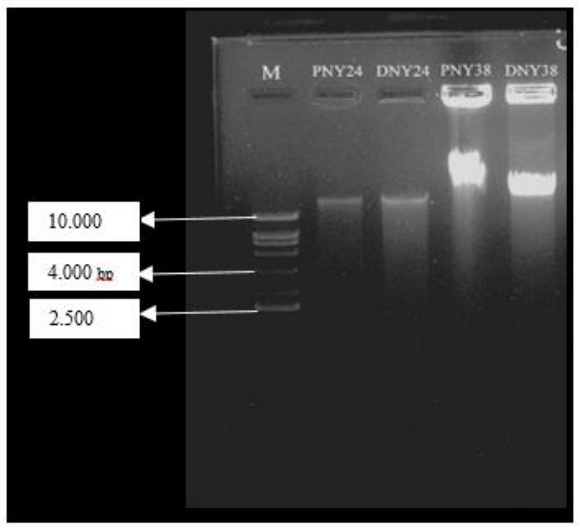

Figure 4. Electrophoresis profile from DNA and plasmid isolation of isolate NY24 and NY38 using Vivantis $1 \mathrm{~kb}$ marker in lane 1. The second and third lane are the electrophoresis result for plasmid and DNA isolation of isolate NY24. Lane four and five are the result of plasmid and DNA isolation for isolate NY38. The result shows there is no existence of extrachromosomal DNA.

Based on the data obtained, there is no indication the presence of extrachromosomal material for the 2 isolates, indicating that the antagonistic activity is solely governed by the chromosome. The research is continued with the molecular identification of the yeasts obtained by performing sequencing reaction that employs primer pairs covering ITS region, which is already known as a universal marker for fungi detection and identification [15]. Figure 6 shows the PCR product of the isolates obtained with length around 500-700 bp.

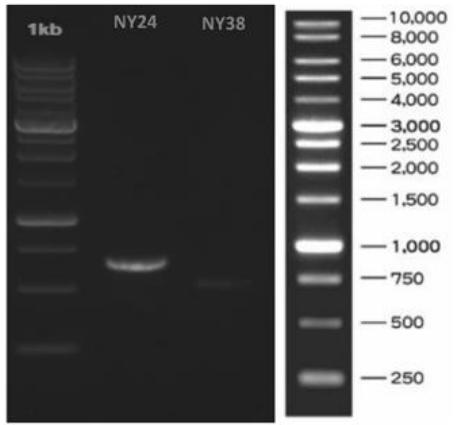

Figure 5. PCR result of NY24 (lane two) and NY38 (lane three) using ITS marker giving rise to an amplicons of about 500-700 bp.

Yeasts with the antagonistic activity could be recovered from diverse insects' digestive tract, showing the biological importance of this microorganism $[16,17]$. It is believed that yeast involved in normal function of the digestive tract, and in return they also benefited as being endosymbiont. The isolation of antagonistic yeast from mosquitoes resulting in 2 isolates with no extrachromosomal materials that is responsible for the killing activity. None of the isolates obtained show the existence of VLE, which are often involve as a coding unit for proteins that kill or inhibit the growth of the sensitive strains. The fact that the 2 isolates obtained have no extra-chromosomal material coincide well when considering their taxonomical position with the already well known VLE-based killler yeast, as already reported elsewhere [7, 14], as can be seen in Figure 7 below.
Phylogenetic analysis shows that isolate NY24 located at the same clade with Microstromatales $\mathrm{sp}$ and Jaminaea sp, at cluster I, with \% identity, query coverage and E-value of $100 \%, 93 \%, 0.0$ and $99 \%$, $98 \%$, and 0.0, respectively (data not shown). According to this, isolate NY24 shows closer relationship to Jaminaea sp as it has better coverage compared to Microstromatales sp. It is reported before the identification of Jaminaea sp from bird's cloacae which implies the adaptability of this yeast with different hosts and for the extreme condition [18]. The tree shows that isolate NY38 is located at the different cluster (cluster II) with NY24; it has close relationship with Candida tropicalis with \% identity and query coverage of $99 \%$, and E-value of 0.0 (data not shown). This result tells us the diversity of yeasts that are found in the mosquitoes from local area in Yogyakarta. 


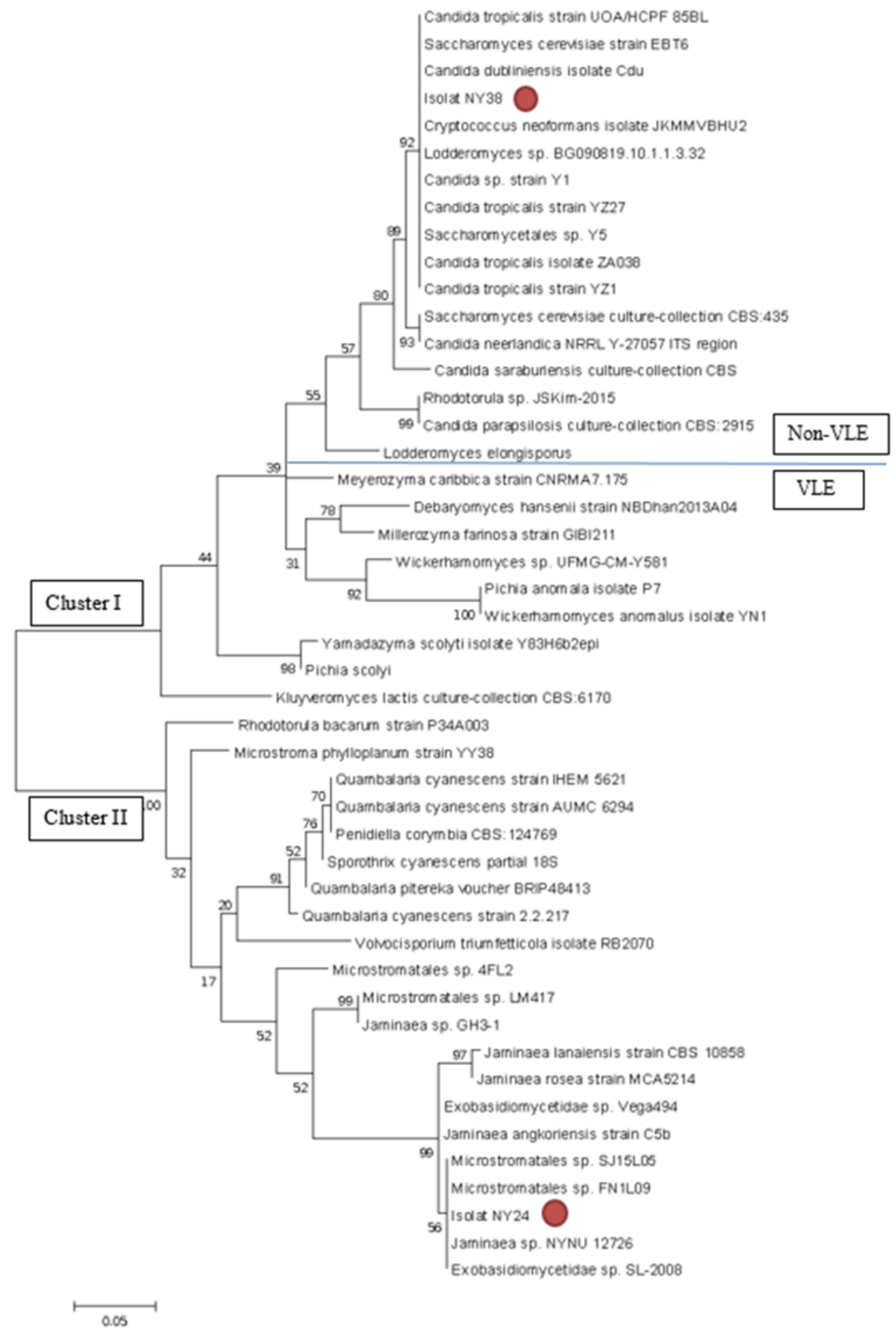

Figure 6. Phylogenetic tree showing the location of isolate NY24 and NY38, and its VLE counterpart.

Another interesting finding is the separation of obtained isolates from Meyerozyma sp, Wickerhamomyces anomalus, Debaryomyces hansenii, and Kluyveromyces lactis which are already known as yeasts able to produce killer toxins [19]. These killer yeasts are known to host what is so called virus-like element (VLE) which are responsible for the coding of killer proteins [14]. The antagonistic assays from our research showed inhibitory effect of our isolates against the sensitive strain, and no indication of the presence of extrachromosomal material, which tell us the consistency of molecular data and antagonistic assay. It is probable that the antagonistic activity 
exert by the isolates obtained are chromosomally encoded. Further researches are need to be done to confirm this suggestion, as well as to check the in

\section{CONCLUSION}

Based on our findings, two yeasts species identified as Jaminaea sp and Candida sp were isolated from local mosquitoes. These yeasts showed the inhibitory activity against the sensitive strain, and ACKNOWLEDGEMENT

Financial support for part of this study from the Dept. of Biology, Faculty of Biotechnology, Universitas Kristen Duta Wacana, Yogyakarta, is gratefully acknowledged

\section{REFERENCES}

[1] Shaw WR and Catteruccia F 2018 Vector biology meets disease control: Using basic research to fight vector-borne diseases. Nature Microbio.

https://www.nature.com/articles/s41564-0180214-7

[2] Ricci I, Valzano M, Ulissi U, Epis S, Cappelli A and Favia G 2012 Symbiotic control of mosquito borne disease, Pathogens and Global Health, 106 380-5

[3] Wang S, Dos-Santos ALA, Huang W, Liu KC, Oshaghi MA, Wei G, Agre P and JacobsLorena, M 2017 Driving mosquito refractoriness to Plasmodium falciparum with engineered symbiotic bacteria. Science, $\mathbf{3 5 7}$ 1399-1405

[4] Cappelli A, Ulissi U, Valzano M, Damiani C, Epis S, Gabrielli M, Conti S, Polonelli L, Bandi C, Favia G and Ricci I. 2014. A Wickerhamomyces anomalus killer strain in the malaria vector Anopheles stephensi. PLoS One, 9, p.e95988

[5] Schneider J, Rupp O, Trost E, Jaenicke S, Passoth V, Goesmann A, Tauch A and Brinkrolf K 2012 Genome sequence of Wickerhamomyces anomalus DSM 6766 reveals genetic basis of biotechnologically important antimicrobial activities. FEMS Yeast Research, 12 382-6

[6] Valzano M, Cecarini V, Cappelli A, Capone A, Bozic J, Cuccioloni M, Epis S, Petrelli D, Angeletti M, Eleuteri A, Favia G and Ricci I 2016 A yeast strain associated to Anopheles mosquitoes produces a toxin able to kill malaria parasites. Malar. J. 15 21-29

[7] Klassen R, Schaffrath R, Buzzini P and Ganter PF 2017 Antagonistic interactions and killer yeasts. DOI: 10.1007/978-3-319-61575-2_9

[8] Perez M, Contreras L, Garnica N, FernándezZenoff M, Farías M, Sepulveda M, Ramallo J vitro and in vivo inhibition of protein produced by the isolates obtained.

this activity is not governed by the extrachromosomal material. Researches are still need to be done to ensure their ability to inhibit the growth of the mosquitoes, either in vitro or in vivo.

and Dib J 2016 Native killer yeasts as biocontrol agents of post-harvest fungal diseases in lemons. PLoS One. 11 p.e0165590

[9] Stam J, Kwakman J, Meijer M and Stuitje A 1986 Efficient isolation of the linear DNA killer plasmid of Kluyveromyces lactis: evidence for location and expression in the cytoplasm and characterization of their terminally bound proteins. Nucl. Ac. Res. 14 6871-84

[10] Larkin MA, Blackshields G, Brown NP, Chenna R, McGettigan PA, McWilliam H, Valentin F, Wallace IM, Wilm A, Lopez R, Thompson JD, Gibson TJ and Higgins DG 2007 Clustal W and Clustal X version 2.0. Bioinformatics. 23 2947-48

[11] Tamura K, Peterson D, Peterson N, Stecher G, Nei M and Kumar S 2011 MEGA5: Molecular evolutionary genetics analysis using maximum likelihood, evolutionary distance, and maximum parsimony methods. Mol. Biol. Evol. 28 2731-39

[12] Ni L and Snyder M 2001 A genomic study of the bipolar bud site selection pattern in Saccharomyces cerevisiae. Mol. Biol. Cell.12 2147-70

[13] Muccilli S and Restuccia C 2015 Bioprotective role of yeasts. Microorganisms. 3 588-611

[14] Satwika D, Klassen R and Meinhardt F 2012 Anticodon nuclease encoding virus-like elements in yeast. Appl. Microbiol. Biotechnol. 96 345-356

[15] Schoch C, et al 2012 Nuclear ribosomal internal transcribed spacer (ITS) region as a universal DNA barcode marker for Fungi. PNAS. 109 6241-46

[16]Boccazzi I, Ottoboni M, Martin E, Comandatore F, Vallone L, Spranghers T, Eeckhout M, Mereghetti V, Pinotti L and Epis S 2017 A survey of the mycobiota associated with larvae of the black soldier fly (Hermetia illucens) reared for feed production. PLoS One, 12 p.e0182533

[17] Gonzalez F 2014 Symbiosis between yeasts and insects. Introductory paper at the Faculty of Landscape Architecture, Horticulture and Crop Production Science. 3: 1-52 
[18] Francesca N, Canale DE, Settanni L and Moschentti G 2012 Dissemination of winerelated yeasts by migratory birds. Env. Microbiol. Rep. 4 105-112

[19] El-Banna AA, El-Shan MA and Shehata MG 2011 Yeasts producing killer toxins : An overview. Alex. J. Food. Sci. Tech. 8 41-53 